\title{
FRACTURAS OSTEOPORÓTICAS DE RAMAS PÉLVICAS: ¿SON UNA LESIÓN BENIGNA?
}

\section{OSTEOPOROTIC PUBIC RAMI FRACTURE: A BENIGN INJURY?}

\author{
Marina Angulo Tabernero ${ }^{1,6}$, Teresa Fernández Letamendi², Begoña Hidalgo Mendía ${ }^{3}$, Julia Ungría \\ Murillo $^{4}$, Belen Gros Bañeres ${ }^{2}$, Nieves Fernández Letamendi ${ }^{5}$, Antonio Peguero Bona ${ }^{1}$
}

\section{Resumen:}

Objetivo: Analizar los pacientes tratados por fractura osteoporótica de ramas pélvicas en nuestro hospital. Métodos: Analizamos retrospectivamente pacientes con fracturas de ramas pélvicas atendidos en el Servicio de Urgencias de nuestro hospital. Los parámetros analizados incluyen datos demográficos, diagnósticos, lesiones asociadas, comorbilidades, autonomía previa, complicaciones y mortalidad al año. Resultados: 60 pacientes (51 mujeres) con una edad media de 83,5 años (rango, 65,1-99) presentaron fractura osteoporótica de ramas pélvicas. 6 pacientes presentaban antecedentes de fractura de ramas pélvicas y 23 de fractura osteoporótica de otra localización. En 27 casos se asociaban a lesiones en otras localizaciones de la pelvis. 3 pacientes asociaban fracturas extrapélvicas y 6 traumatismos craneoencefálicos. 41 pacientes experimentaron complicaciones de algún tipo. Se requirió ingreso hospitalario en 8 casos, con una estancia media de 18 días. La mortalidad al año fue de 13,3\%.

Conclusiones: Las fracturas osteoporóticas de ramas pélvicas afectan predominantemente a mujeres con numerosas comorbilidades. Muchas son las complicaciones descritas en estos pacientes con una mortalidad anual elevada. Es recomendable un adecuado tratamiento analgésico que permita una movilización precoz del paciente tras descartar lesiones asociadas en la pelvis.

Palabras clave: fractura osteoporótica; fractura de rama pélvica; anciano; morbilidad.

\section{Abstract:}

Objective: We analized patients treated for osteoporotic rami fractures in our hospital.

Methods: We examined the records of patients presenting to the emergency department with osteoporotic low-impact rami fractures. We describe demographic, previously ambulatory ability, diagnosis, associated injuries, comorbility, complications and 1 year mortality.

Results: 60 patients (51 women) with a mean age of 83,5 (range, 65,1-99) years had osteoporotic rami fracture. Six patients had previously a rami fracture and 23 a osteoporotic fracture. 27 patients associated other pelvic fracture. Three pacientes had other extrapelvic fracture and six a craneoencephalic trauma. 41 patients had any complication at follow up. Eigth patients $(13,3 \%)$ were admitted to hospital with a median length of stay in the hospital of 18 days. Eight $(13,3 \%)$ patients died within 1 year of injury.

Conclusions: Low-impact rami fractures affect predominantly elderly women with pre-existing comorbidities. A substantial amount of complications are described in this fractures with a high 1 year mortality. Early rehabilitation therapy with adequate analgesic therapy should be recommended.

Keywords: osteoporotic fracture; osteoporotic rami fracture; elderly; morbility.

\footnotetext{
1 Servicio de Traumatología y Ortopedia. Hospital Universitario Miguel Servet, Zaragoza, España

2 Servicio de Urgencias. Hospital Universitario Miguel Servet, Zaragoza, España

3 Servicio de Rehabilitación. Hospital Universitario Miguel Servet, Zaragoza, España

4 Servicio de Anestesiología y Reanimación. Hospital Universitario Miguel Servet, Zaragoza, España

5 Servicio de Geriatría. Hospital Universitario Miguel Servet, Zaragoza, España

6 Email de contacto: marinaangulo@hotmail.com
} 


\section{Introducción}

Las fracturas de pelvis representan entre el 3 y el $8 \%$ de las lesiones esqueléticas traumáticas ${ }^{[1]}$. Las fracturas osteoporótica de ramas (FOR) tras traumatismo de baja energía son frecuentes en ancianos. Su incidencia en la población general es de 37/100000 pacientes. Esta incidencia aumenta con la edad y alcanza su pico máximo en mujeres mayores de 85 años (450/100000 pacientes/año) ${ }^{[2]}$.

Debido al envejecimiento de la población es una patología en aumento ${ }^{[3,4]}$, atendida con cada vez mas frecuencia en los servicios de urgencias. La mayoría de fracturas de pelvis en los ancianos son tratadas de manera conservadora con analgésicos y reposo en descarga suponiendo una alta carga socioeconómica y perdida de autonomía para el paciente y familiares ${ }^{[5]}$.

Es bien conocida la epidemiologia y pronostico de otras fracturas osteoporóticas del anciano, como las fracturas de cadera ${ }^{[6]}$, pero existen pocos datos sobre las características y los resultados funcionales de los pacientes con FOR. Aunque las fracturas osteoporóticas de cadera son tres veces más frecuentes que las de ramas pélvicas, el incremento de la mortalidad en ambos tipos es similar, aumentando entre el 2,0 a 2,5 veces $^{[6]}$.

A pesar de tratarse de fracturas de baja energía en ocasiones puede poner en peligro la vida del paciente por hipovolemia por sangrado, lesiones de estructuras pélvicas, complicaciones sistémicas,... Además del riesgo que la inmovilización puede suponer en este grupo de pacientes de edad avanzada con múltiples comorbilidades asociadas.

El objetivo de este estudio es conocer las morbilidad y mortalidad al año de este tipo de fractura.

\section{Pacientes y Métodos}

Este estudio se ha realizado en el Hospital Universitario Miguel Servet, hospital de referencia del Sector 2 de Zaragoza (España). El número de habitantes que dependen de nuestro hospital es de aproximadamente 400.000 habitantes.

En nuestra ciudad la proporción de población mayor de 65 años se ha visto incrementada durante los últimos años, alcanzando ya el 17,7 \% de la población de la ciudad de Zaragoza (119.353 habitantes) ${ }^{[7]}$.

Se registraron de manera retrospectiva los pacientes atendidos en el Servicio de Urgencias con el diagnostico de esguince de pelvis, fractura de pelvis, fractura de sacro y fractura de ramas entre el 1 de enero y el 31 de diciembre de 2012.

Los criterios de inclusión fueron pacientes de 65 años o más con diagnostico de fractura de ramas pélvicas tras traumatismo de baja energía, definida como caída desde una altura menor de un metro o en ausencia de traumatismo conocido ${ }^{[8]}$. Se excluyeron del estudio las fracturas debidas a traumatismos de alta energía como accidentes de tráfico, caídas desde más de un metro de altura y fracturas patológicas.

Se realizó una revisión de la historia clínica electrónica de los pacientes, base de datos donde se registran la información de atención primaria, especializada y hospitalaria de todos los hospitales de la Comunidad Autónoma de Aragón, para obtener información demográfica, comorbilidades, autonomía previa, tratamientos habituales, tiempo de estancia en urgencias, pruebas diagnosticas realizadas, lesiones asociadas, tratamiento y recomendaciones al alta, ingresos (urgencias $u$ hospitalarios), complicaciones y mortalidad al año.

Se utilizó estadística descriptiva para analizar los datos con el programa SPSS en su Versión 20.0 para Mac.

\section{Resultados}

Durante el periodo de estudio un total de 184 pacientes fueron diagnosticados de fractura pélvica. Se excluyeron 124 pacientes del estudio por ser menores de 65 años o presentar lesiones de alta energía u otras fracturas pélvicas. Los 60 casos restantes cumplían la definición de FOR. Los grupos de fracturas por traumatismo de alta y baja energía se diferenciaron en términos demográficos. El grupo de alta energía fue predominantemente masculino (73\%) y con pacientes más jóvenes, con una edad media de 37,3 (rango, 16 - 78) años, mientras que el grupo de baja energía fue predominantemente femenino (85\%) con una edad media de 83,5 (rango, 65,1 - 99) años.

Las características de los pacientes con FOR se reflejan en la Tabla 1. La caída fue el mecanismo de lesión principal en $58(96,7 \%)$ pacientes y no se asoció a traumatismo conocido en $2(3,3 \%)$ casos. El lugar más frecuente el traumatismo fue la casa o residencia habitual en 35 (58,3\%) de los casos. 
Tabla 1: Características de los 60 pacientes con FOR.

\begin{tabular}{|lc|}
\hline \multicolumn{1}{|c|}{ Características } & $\begin{array}{c}\text { Numero (\%) } \\
\text { de pacientes }\end{array}$ \\
\hline Sexo & \\
Mujer & $51(85)$ \\
Varón & $9(15)$ \\
\hline Edad media (rango) & $83,5(65,1-99)$ \\
\hline Estado ambulatorio previo & \\
Autónoma & $25(41,7)$ \\
Apoyo unilateral & $20(33,3)$ \\
Apoyo Bilateral & $1(1,7)$ \\
Andador & $5(8,3)$ \\
Silla de ruedas & $2(3,3)$ \\
No especifica & $7(11,7)$ \\
\hline Mecanismo de fractura & \\
Caída & $58(96,7)$ \\
No traumatismo conocido & $2(3,3)$ \\
\hline Lugar de la caída & $35(58,3)$ \\
Casa - Residencia & $9(15)$ \\
Calle & $16(26,7)$ \\
No registrado & $35(58,3)$ \\
Tiempo desde la caída a llegada al SU & $11(18,4)$ \\
Menor de 8 horas & $10(16,7)$ \\
8 - 24 horas & $4(6,7)$ \\
24 horas - 7 días & $32(53,3)$ \\
Mayor de 7 días & $27(45)$ \\
\hline Lateralidad pelvis & $1(1,7)$ \\
Derecho & $27(45)$ \\
Izquierdo & $22(36,7)$ \\
Bilateral & $3(5)$ \\
Lesiones pélvicas asociadas & $1(1,7)$ \\
Acetábulo & $1(1,7)$ \\
Sacroilíaca & $6(10)$ \\
Ala sacra & $2(3,3)$ \\
Ilion & $1(1,7)$ \\
TCE & $1(1,7)$ \\
Fractura de radio distal & \\
Fractura de humero proximal & \\
\hline
\end{tabular}

TCE (Traumatismo cráneo-encefálico)

Las comorbilidades y tratamientos se reflejan en la Tabla 2. Solo un paciente no tenía comorbilidades ni tratamientos asociados. Entre los antecedentes traumatológicos de los pacientes encontramos que 6 $(10 \%)$ habían sufrido una FOR previamente y $23(38,3 \%)$ algún otro tipo de fractura osteoporótica (fractura de radio distal, fémur proximal, humero proximal o vertebral). Respecto a tratamientos habituales destacar que $30(50 \%)$ pacientes tomaban de manera habitual algún tratamiento que favorecía el sangrado y solo $20(33,3 \%)$ estaban en tratamiento para la osteoporosis en el momento de la nueva fractura.

Tabla 2: Comorbilidades y tratamientos de los 60 pacientes con fractura osteoporótica de ramas pélvicas.

\begin{tabular}{|lc|}
\hline \multicolumn{1}{|c|}{ Características } & $\begin{array}{c}\text { Número (\%) de } \\
\text { pacientes }\end{array}$ \\
\hline Comorbilidades asociadas & $59(98,3)$ \\
Hipertensión arterial & $42(70)$ \\
Fractura osteoporótica previa & $29(48,3)$ \\
Enf. cardiovascular o arritmia & $18(30)$ \\
Enf. neurológica & $17(28,3)$ \\
Artrosis & $17(28,3)$ \\
Depresión - Ansiedad & $16(26,7)$ \\
Dislipemia & $15(25)$ \\
Diabetes & $14(23,3)$ \\
Osteoporosis & $14(23,3)$ \\
Enfermedad respiratoria & $12(20)$ \\
Enfermedad endocrina & $10(16,6)$ \\
Demencia & $8(13,4)$ \\
\hline
\end{tabular}




\begin{tabular}{|c|c|}
\hline Enfermedad Hematológica & $4(6,7)$ \\
\hline Insuficiencia Renal & $4(6,7)$ \\
\hline Sin comorbilidades & $1(1,7)$ \\
\hline Tratamiento antiagregante/anticoagulante & $30(50)$ \\
\hline Antiagregantes & $19(31,7)$ istepis \\
\hline Acenocumarol & $7(11,7)$ \\
\hline НВРМ & $2(3,3)$ \\
\hline Doble antiagregación & $1(1,7)$ \\
\hline Antiagregante + anticoagulante & $1(1,7)$ \\
\hline Tratamiento Osteoporosis & $20(33,3)$ \\
\hline Calcio y Vitamina D & $11(18,3)$ \\
\hline Bifosfonatos & $7(11,7)$ \\
\hline SERM & $1(1,7)$ \\
\hline Estroncio & $1(1,7)$ \\
\hline
\end{tabular}

La fractura más frecuente fue la que implicaba a ambas ramas ilio e isquiopubiana en $50(83,3 \%)$ casos. La afectación de una rama aislada se objetivó en 9 (15\%) casos y de ellos, la más frecuentemente fracturada fue la isquiopubiana en $6(10 \%)$ casos. Un caso fue diagnosticado de FOR bilaterales. 27 (45\%) pacientes tenían lesiones asociadas en otras localizaciones de la pelvis, siendo la afectación del acetábulo la más frecuente en $22(36,7 \%)$ de ellos, menos frecuentemente, se observaron las lesiones de la articulación sacroilíaca, fracturas del ala sacra o del ilion (3 (5\%), 1 (1,7\%) y $1 \quad(1,7 \%)$ respectivamente). De manera asociada a la caída se objetivaron fracturas extrapelvicas en $4(6,6 \%)$ casos y traumatismo craneoencefálico en $6(10 \%)$.

En 58 (96,7\%) pacientes fue necesario estudio radiológico de la pelvis para el diagnostico. En 9 (15\%) casos el estudio inicial se completo con una tomografía computarizada (TC) por dudas radiológicas o sospecha de lesiones pélvicas asociadas. En 18 (30\%) pacientes se realizo analítica de sangre con unos valores medios de hemoglobina y hematocrito de 12,6 (rango, 9,6-14,5) g/dl y 37,5 (rango, 31,1-45,2) \% respectivamente. Estas determinaciones se repitieron tras el periodo de observación en urgencias en 5 $(8,3 \%)$ casos, observando un descenso de la hemoglobina y del hematocrito a 10,3 (rango, 7,6-12,5) g/dl y 30,6 (rango, 22,8-35) \% respectivamente.

Fue necesaria la administración de analgésicos por via parenteral en el Servicio de Urgencias a 25 $(41,7 \%)$ pacientes. En 15 (25\%) casos se emplearon anti-inflamatorios no esteroideos y en otros 9 (15\%) pacientes opioides menores asociados o no al primer escalón de la OMS.

La estancia media en el Servicio de Urgencias fue de 390 (rango, 40-3270) minutos. Permanecieron en observación en urgencias $10(16,7 \%)$ pacientes como paso previo al alta o al ingreso. Durante este periodo $2(3,3 \%)$ pacientes precisaron de la transfusión de concentrados de hematíes por el sangrado. Fueron hospitalizados $8(13,3 \%)$ pacientes a cargo del Servicio de Traumatología con una estancia media de 18 (rango, 7-28) días. Una de las pacientes ingresadas fue intervenida quirúrgicamente realizándose un atornillado percutáneo retrogrado de la rama ilio-pubiana permitiendo carga precoz, menor demanda de analgésicos y sin presentar complicaciones asociadas.

La salida del Servicio de Urgencias fue en ambulancia convencional en 47 (90,4\%) de los 52 pacientes dados de alta desde dicho servicio. Entre las recomendaciones al alta se encontraban la necesidad de reposo en descarga y diferentes escalones analgésicos en todos los casos. Se indicó profilaxis con heparinas de bajo peso molecular en $46(76,6 \%)$ casos. El número medio de recetas para cada paciente fue de 3,2 (rango, 0-5). Entre los analgésicos pautados se encontraban en 56 recetas de fármacos del primer escalón de la OMS (paracetamol, metamizol o AINES), 11 del segundo escalón (opioides menores) y 4 del tercer escalón (opioides mayores).

De los 60 pacientes diagnosticados de FOR se registraron complicaciones en $41(68,3 \%)$ casos, muchas de ellas solventadas desde los centros de atención primaria o durante la revisión en la consulta de traumatología sin requerir nueva asistencia en urgencias. La principal complicación fue el mal control del dolor en $23(38,3 \%)$ casos. Sin embargo, 14 (23,3\%) pacientes regresaron al Servicio de Urgencias por segunda vez dentro del mes siguiente a la fractura requiriendo ingreso 5 de ellos por complicaciones directamente atribuibles a la fractura. 7 pacientes requirieron una tercera asistencia de urgencias por lo que fueron hospitalizados 5 de ellos. En la Tabla 3 se detallan las complicaciones encontradas y su frecuencia.

Tabla 3: Complicaciones y frecuencia de los pacientes con FOR.

\begin{tabular}{|lc|}
\hline Complicaciones & Numero $(\%)$ pacientes \\
\hline Mal control del dolor & $23(38,3)$ \\
\hline
\end{tabular}




\begin{tabular}{|lc|}
\hline Alteraciones analíticas & $10(16,7)$ \\
Infección de Orina & $5(8,3)$ \\
Delirium & $5(8,3)$ \\
Neumonía & $4(6,7)$ \\
Episodio trombótico (TEP - TVP) & $2(3,3)$ \\
Íleo paralitico & $1(1,7)$ \\
\hline
\end{tabular}

Durante la estancia hospitalaria una paciente falleció debido a una infección respiratoria. Tras la revisión de la historia clínica electrónica se recogieron $7(11,7 \%)$ casos más de pacientes que fallecieron tras el primer año de la fractura, lo que supone una mortalidad al año de la fractura del 13,3\% (8 casos).

\section{Discusión}

Las fracturas osteoporóticas son cada vez más frecuentes en los Servicios de Urgencias debido al envejecimiento de la población. Son muy estudiadas las fracturas relacionadas con la fragilidad ósea a nivel de fémur proximal o columna vertebral, sin embargo pocos son los trabajos que analicen la morbimortalidad de las fracturas de ramas pélvicas.

Las fracturas de ramas, lesión ósea más frecuente de la pelvis, han sido consideradas clásicamente como una fractura benigna, tratada mediante reposo y analgésicos habituales ${ }^{[9]}$. Sin embargo, en los últimos años su estudio están ganando importancia debido a su aumento de frecuencia en los Servicios de Urgencias. El seguimiento en la mayoría de los centros hospitalarios de estas fracturas se realiza desde los Servicios de Traumatología pero varios trabajos proponen, al igual que en las fracturas osteoporóticas de fémur proximal, el manejo conjunto con los Servicios de Geriatría en unidades multidisciplinares ${ }^{[2,4,10]}$.

A pesar de tratarse de fracturas de baja energía y clásicamente clasificarse como estables (fracturas Tipo A según la clasificación de la AO/ASIF) [11], las fracturas osteoporóticas de ramas pélvicas, se acompañan en un alto porcentaje de casos de lesiones en la articulación sacroilíaca, fracturas de ilion o fracturas por compresión en el sacro ${ }^{[12,13]}$. Lau y Leung ${ }^{[14]}$ realizaron una tomografía axial computarizada a 37 pacientes con fractura osteoporóticas de ramas pélvicas con una edad media de 85 años, observaron que en 22 casos (59\%) se asociaban fracturas en los elementos posteriores de la pelvis, con lo que proponen la realización del TAC de manera rutinaria para el diagnostico de este tipo de fracturas. Cosker et al. ${ }^{[15]}$ realizaron una $\mathrm{RMN}$ a 50 fracturas osteoporóticas de ramas pélvicas tras una traumatismo de baja energía, observando 45 fracturas de sacro. En estos trabajos destacan también la alta correlación del dolor posterior durante la exploración física con la presencia de lesiones asociadas a este nivel y atribuyen la persistencia de la clínica dolorosa más allá de la consolidación de la fractura de ramas a esta lesión, la mayoría de las veces inadvertida e infratratada.

En nuestro caso los 5 pacientes con afectación de los elementos posteriores fueron diagnosticados mediante TC, realizado por la sospecha clínica de lesiones asociadas. Creemos que en nuestra revisión esta lesión puede estar infradiagnosticada ya que más de dos terceras partes de las fracturas sacras 0 lesiones sacroiliacas no son visibles en la radiografía simple ${ }^{[16]}$.

También muy es frecuente la afectación acetabular. Dodge y Brison ${ }^{[17]}$ estudiaron 77 fracturas de baja energía con una edad media de 81 años encontrando un $22 \%$ de fracturas de cotilo. En nuestra revisión se observó que en el $36.7 \%$ de las fracturas el trazo comprometía el cotilo. Este diagnóstico en varios casos se realizo por las sucesivas radiografías de control en la consulta, observándose artrosis postraumática coxofemoral en dos de los pacientes.

Muchas son las posibles complicaciones asociadas descritas en estas lesiones. Por un lado observamos las relacionadas directamente con la fractura, como la anemia por sangrado, las lesiones de estructuras viscerales o vasculares vecinas y la pseudoartrosis entre otras, pero en otros casos es consecuencia de su tratamiento mediante inmovilización y analgesia, que para los pacientes con numerosas comorbilidades puede representar un serio peligro.

Las complicaciones hemorrágicas debidas a la lesión de la arteria corona mortis (conexión entre el sistema iliaco externo y el obturador que pasa sobre la rama iliopubiana ${ }^{[18]}$ ) son las más estudiadas en la bibliografía y su tratamiento pasa por la embolización del vaso sangrante ${ }^{[19-21]}$. Esta lesión está descrita en fracturas desplazadas y no desplazadas e indistintamente del mecanismo de lesión (alta o baja energía). El tiempo hasta la aparición de los primeros síntomas de shock hipovolémico descritos en la bibliografía varía desde los 30 minutos a las 6 horas tras la fractura ${ }^{[19]}$. Se ha propuesto este plazo de tiempo como periodo mínimo de observación, especialmente en casos con alto riesgo hemorrágico como son los pacientes de edad avanzada, en tratamiento antiagregante o anticoagulante y con enfermedades con coagulopatías asociadas ${ }^{[19]}$. En una revisión de los casos descritos en la literatura, la única característica común de todos los pacientes con esta complicación es la lesión de la rama superior o 
iliopubiana, pudiendo ser la única rama fracturada ${ }^{[19]}$. Aunque el diagnostico de certeza es mediante la arteriografía se han descrito signos indirectos de hemorragia en las radiografías simples de pelvis que pueden hacer sospechar la lesión ${ }^{[21]}$. En nuestro estudio el $41,7 \%$ de los pacientes acudieron a urgencias transcurridas más de 8 horas del traumatismo habiendo ya cumplido el periodo mínimo de vigilancia recomendado. En 2 casos que permanecieron en observación con factores de riesgo de sangrado fue necesaria la transfusión de concentrados de hematíes por la hemorragia de la fractura asociada a valores bajos de hemoglobina y hematocrito previos, ambos cuadros se resolvieron sin complicaciones.

El tratamiento de estas fracturas se debe orientar a la movilización temprana y el control del dolor, que es la principal complicación. El manejo por lo general es conservador con descarga, analgesia y tromboprofilaxis durante las primeras semanas. Algunos autores, para evitar las complicaciones debidas a la inmovilización, proponen una alta dosis analgésica que permita la carga y la deambulación casi inmediata ${ }^{[22]}$. Otros optan por el tratamiento quirúrgico mediante el atornillado percutáneo de la rama iliopubiana, que en nuestra revisión se realizo a una paciente permitiendo el inicio inmediato de la movilización y mejorando el control del dolor ${ }^{[23]}$. La "ramoplastia", tratamiento más novedoso, consiste en la inyección percutánea de cemento poli-metil-metacrilato que logra una mejoría inmediata del dolor ${ }^{[24,25]}$ al igual que se hace en fracturas osteoporóticas de otras localizaciones, como las vertebrales.

A pesar de ser producidas por un trauma menor y su concepto de benignidad, la mortalidad de las fracturas osteoporóticas de ramas pélvicas no es despreciable. En nuestra revisión encontramos una mortalidad al año del 13,3\%, misma cifra que Hill et al. ${ }^{[4]}$ y similar a Taillandier et al. ${ }^{[5]}, 14,3 \%$. Otros estudios de características similares observan datos muy variables. Morris et al. ${ }^{[2]}$ registraron una mortalidad al año del 27\%, Dodge \& Brison ${ }^{[17]}$ del $17 \%$ y Koval et al. ${ }^{[9]}$ del 9,5\%. Estos son datos muy similares a la mortalidad anual observada en las fracturas de cadera, cuyas cifras son del 14 al $36 \%{ }^{[26]}$.

Para el adecuado manejo de este tipo de fracturas en urgencias creemos que es necesaria la elaboración de un protocolo en el que se plantee un periodo mínimo de observación de las fracturas de 6-8 horas en casos de aumento del riesgo de sangrado con controles analíticos durante este periodo y las pruebas diagnosticas a realizar ante un paciente con fractura de ramas para confirmar la fractura, determinar su desplazamiento o descartar lesiones asociadas de otros elementos de la pelvis. Pueden ser de utilidad en estos casos, previo a la realización del TAC, diferentes proyecciones de la pelvis además de la AP, como son la proyección inlet y oulet ${ }^{[27]}$.

\section{Bibliografía}

[1] Pohlemann T, Tscherne H, Baumgärtel F, Egbers HJ, Euler E, Maurer F, et al. Pelvic fractures: epidemiology, therapy and long-term outcome. Overview of the multicenter study of the Pelvis Study Group. Unfallchirurg 1996;99:160-7.

[2] Morris RO, Sonibare A, Green DJ, Masud T. Closed pelvic fractures: characteristics and outcomes in older patients admitted to medical and geriatric wards. Postgrad Med J 2000;76:646-50.

[3] Kannus P, Palvanen M, Niemi S, Parkkari J, Järvinen M. Epidemiology of osteoporotic pelvic fractures in elderly people in Finland: sharp increase in 1970-1997 and alarming projections for the new millennium. Osteoporos Int J Establ Result Coop Eur Found Osteoporos Natl Osteoporos Found USA 2000;11:443-8. doi:10.1007/s001980070112.

[4] Hill RM, Robinson CM, Keating JF. Fractures of the pubic rami. Epidemiology and five-year survival. J Bone Joint Surg Br 2001;83:1141-4.

[5] Taillandier J, Langue F, Alemanni M, Taillandier-Heriche E. Mortality and functional outcomes of pelvic insufficiency fractures in older patients. Jt Bone Spine Rev Rhum 2003;70:287-9.

[6] Browner WS, Pressman AR, Nevitt MC, Cummings SR. Mortality following fractures in older women. The study of osteoporotic fractures. Arch Intern Med 1996;156:1521-5.

[7] Instituto Aragonés de Estadística - Departamentos y Organismos Públicos - Gobierno de Aragón n.d. http://www.aragon.es/iaest (accessed January 15, 2015).

[8] Cummings SR, Melton LJ. Epidemiology and outcomes of osteoporotic fractures. Lancet 2002;359:1761-7. doi:10.1016/S0140-6736(02)08657-9.

[9] Koval KJ, Aharonoff GB, Schwartz MC, Alpert S, Cohen G, McShinawy A, et al. Pubic rami fracture: a benign pelvic injury? J Orthop Trauma 1997;11:7-9.

[10] Mears SC, Berry DJ. Outcomes of displaced and nondisplaced pelvic and sacral fractures in elderly adults. J Am Geriatr Soc 2011;59:1309-12. doi:10.1111/j.1532-5415.2011.03455.x.

[11] Isler B, Ganz R. Classification of pelvic ring injuries. Injury 1996;27 Suppl 1:S - A3-12.

[12] Kanberoglu K, Kantarci F, Cebi D, Yilmaz MH, Kurugoglu S, Bilici A, et al. Magnetic resonance imaging in osteomalacic insufficiency fractures of the pelvis. Clin Radiol 2005;60:105-11. doi:10.1016/j.crad.2004.04.021.

[13] Renner JB. Pelvic insufficiency fractures. Arthritis Rheum 1990;33:426-30.

[14] Lau T, Leung F. Occult posterior pelvic ring fractures in elderly patients with osteoporotic pubic rami fractures. J Orthop Surg Hong Kong 2010;18:153-7. 
[15] Cosker TDA, Ghandour A, Gupta SK, Tayton KJJ. Pelvic ramus fractures in the elderly: 50 patients studied with MRI. Acta Orthop 2005;76:513-6. doi:10.1080/17453670510044634.

[16] Finiels H, Finiels PJ, Jacquot JM, Strubel D. [Fractures of the sacrum caused by bone insufficiency. Metaanalysis of 508 cases]. Presse Médicale Paris Fr 1983 1997;26:1568-73.

[17] Dodge G, Brison R. Low-impact pelvic fractures in the emergency department. CJEM 2010;12:509-13.

[18] Tornetta P, Hochwald N, Levine R. Corona mortis. Incidence and location. Clin Orthop 1996:97-101.

[19] Kong W-M, Sun C-K, Tsai I-T. Delayed presentation of hypovolemic shock after a simple pubic ramus fracture. Am J Emerg Med 2012;30:2090.e1-4. doi:10.1016/j.ajem.2011.12.030.

[20] Macdonald DJM, Tollan CJ, Robertson I, Rana B, Rana BS. Massive haemorrhage after a low-energy pubic ramus fracture in a 71-year-old woman. Postgrad Med J 2006;82:e25. doi:10.1136/pgmj.2006.047381.

[21] Mouzopoulos G, Tzurbakis M, Mouzopoulos D, lerodiakonou V, Tsembeli A, Georgilas I. Massive haemorrhage due to minimally displaced pubic ramus fracture. Eur $J$ Emerg Med Off $J$ Eur Soc Emerg Med 2009;16:271-2. doi:10.1097/MEJ.0b013e3282f20e0c.

[22] Krappinger D, Struve P, Schmid R, Kroesslhuber J, Blauth M. Fractures of the pubic rami: a retrospective review of 534 cases. Arch Orthop Trauma Surg 2009;129:1685-90. doi:10.1007/s00402-009-0942-5.

[23] Beall DP, Datir A, D'Souza SL, D'Souza LS, Gunda D, Morelli J, et al. Percutaneous treatment of insufficiency fractures : principles, technique and review of literature. Skeletal Radiol 2010;39:117-30. doi:10.1007/s00256009-0722-x.

[24] Beall DP, D'Souza SL, Costello RF, Prater SD, Van Zandt BL, Martin HD, et al. Percutaneous augmentation of the superior pubic ramus with polymethyl methacrylate: treatment of acute traumatic and chronic insufficiency fractures. Skeletal Radiol 2007;36:979-83. doi:10.1007/s00256-007-0313-7.

[25] Kamysz J, Rechitsky M. Pubic bone cement osteoplasty for pubic insufficiency fractures. J Vasc Interv Radiol JVIR 2008;19:1386-9. doi:10.1016/j.jvir.2008.05.026.

[26] Carpintero P, Caeiro JR, Carpintero R, Morales A, Silva S, Mesa M. Complications of hip fractures: A review. World J Orthop 2014;5:402-11. doi:10.5312/wjo.v5.i4.402.

[27] Gänsslen A, Krettek C. Retrograde transpubic screw fixation of transpubic instabilities. Oper Orthop Traumatol 2006;18:330-40. doi:10.1007/s00064-006-1181-3. 